\title{
Matrix Metalloproteinases In Cancer Biology: A Review
}

Ibrahim $W N^{a^{*}}$, Abdull Rasad MSB $B^{b}$ \& Doolanea $A A^{c}$

${ }^{a}$ Department of Basic Medical Sciences for Nursing, Faculty of Nursing, International Islamic University Malaysia, Kuantan Pahang, Malaysia.

${ }^{\mathrm{b}}$ Department of BioMedical Sciences, Faculty of Allied Health Sciences, International Islamic University Malaysia, Kuantan Pahang, Malaysia.

' Department of Pharmaceutical technology, Faculty of Pharmacy, International Islamic University Malaysia, Kuantan Pahang, Malaysia.

\section{ABSTRACT}

Matrix metalloproteinases (MMPs) are a group of proteinases that are involved with the enzymatic breakdown of the extracellular matrix and basement membrane components. These enzymes are important in regulating vital physiological functions such as embryonic development, wound healing and tissue remodelling. However, several disorders may result from the exaggerated function of these enzymes such as ulcers, rheumatoid arthritis and invasive tumours. In tumours, the effect of these enzymes is not limited to invasion as it was traditionally believed but it also extends to the other oncogenic hallmark processes such as proliferation, angiogenesis, epithelial-mesenchymal transition and evasion from apoptosis. Therefore, it is essential to thoroughly understand the molecular mechanisms involved in these enzymes in cancerous tissue based on recent literature. Several reviews have highlighted the function of these enzymes in malignancies however the aim of this was to provide more recent overview to their role in malignant transformation and progression and in a briefer approach summarizing the complex molecular pathways. Online databases such as PubMed, Google scholar, Web of Science and MEDLINE were used to identify relevant articles. This approach would assist researchers by providing a list of the potential molecular targets in the sequence of changes related to these enzymes. This might help in designing a safer and a more specific targeted treatment for patients with cancer.

KEYWORDS: Matrix Metalloproteinases, cancer, epithelial mesenchymal transition, invasion, metastasis

\section{INTRODUCTION}

Matrix metalloproteinases (MMPs) are extracellular zinc-dependent endopeptidases that have been found in vertebrates, invertebrates and plants. These enzymes are characterized by their dependence on metal ions as cofactors and their ability to degrade all components of the extracellular matrix (ECM). ${ }^{1}$ MMPs are classified by their structure and the type of substrate into several sub-groups as shown in Figure 1. Subclasses include collagenases which are capable of degrading most of fibrillar collagen, gelatinases that degrades collagen type I and IV, stromelysins which have a similar

Corresponding Author

Asst. Prof. Dr Wisam Nabeel Ibrahim

Department of Basic Medical Sciences

Faculty of Science Building (Biological Sciences)

International Islamic University Malaysia

25200 Kuantan Pahang.

Tel No : 09-570 7307

Email :wisam@iium.edu.my structure to collagenases but with different substrate specificity, matrilysins that degrades collagen type IV and membrane type MMPs (MT-MMPs) which are capable of degrading fibrillar collagen type . $^{1-4}$

In normal physiological conditions, the functions of these enzymes is important and is tightly regulated to orchestrate a balanced reformation of the ECM in conditions such as in tissue remodelling, embryonic development and in tissue repair. ${ }^{5}$ However, when the normal levels of these enzymes are exceeded, it may result in number of pathological conditions characterized by an accelerated breakdown of extracellular matrix causing intense and destructive inflammatory disorders such as rheumatoid arthritis, atherosclerosis, osteoarthritis and in malignancies. ${ }^{6,7}$ The unbalanced function of MMPs in tumours depends on a complex interactions between cancer cells, ECM and cancer stromal cells causing an accelerated remodelling of the ECM. ${ }^{2}$ 


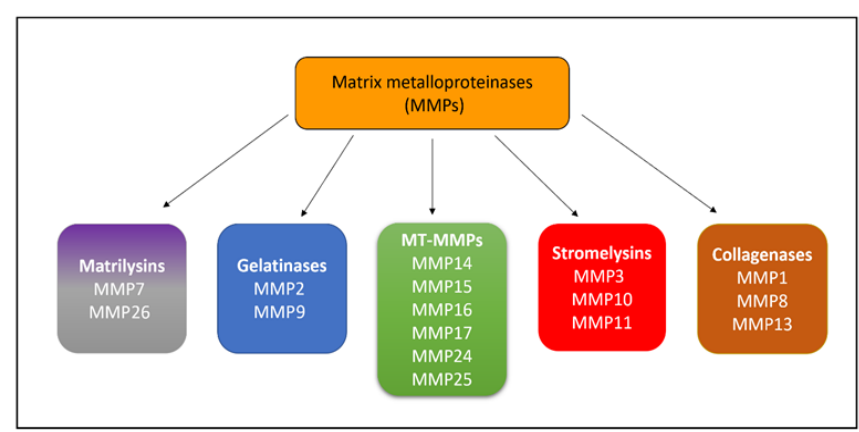

Figure 1 Classification of matrix metalloproteinases

This review describes the roles and the molecular mechanisms involved in the action of MMPs within the tumour microenvironment in a more practical approach that might assist researchers in developing future treatments for cancer patients.

\section{METHODOLOGY}

This narrative review was conducted by searching online databases including PubMed, Google scholar, Web of Science and MEDLINE for articles and review papers with titles including Matrix metalloproteinases or MMPs and cancer, tumour or malignancy. Any article that is related to pathologies other than malignancies or was dated before 2010 unless no update was found to be excluded from this review. The identified review papers were scrutinized for details that were validated and updated according to the most recent findings. Articles with contradictory findings were carefully evaluated for credibility before being included in this review. Therefore, this review provides enough evidences about the different roles of MMPs regarding the development of tumour tissue that is explained in a systematic and brief order.

\section{Effects of matrix metalloproteinases on tumour pathogenesis}

Generally, MMPs contribute in the carcinogenesis process by breaking the physical barriers surrounding the cancer cells and enhancing migration of cancer cells through facilitation of invasion and metastasis processes. ${ }^{16}$ However, the function of these enzymes is not limited to the breakdown of ECM; it can extend to facilitate the progression of cancer cells through other processes such as EMT, proliferation, angiogenesis and evasion from apoptosis as shown in Figure 2. ${ }^{16-18}$ Most of these processes are triggered by degrading the different components within the ECM via the proteolytic function of these enzymes that allows release or activation of different growth factors, or shedding of adhesion molecules that modulates the status of cellular attachments. ${ }^{2}$

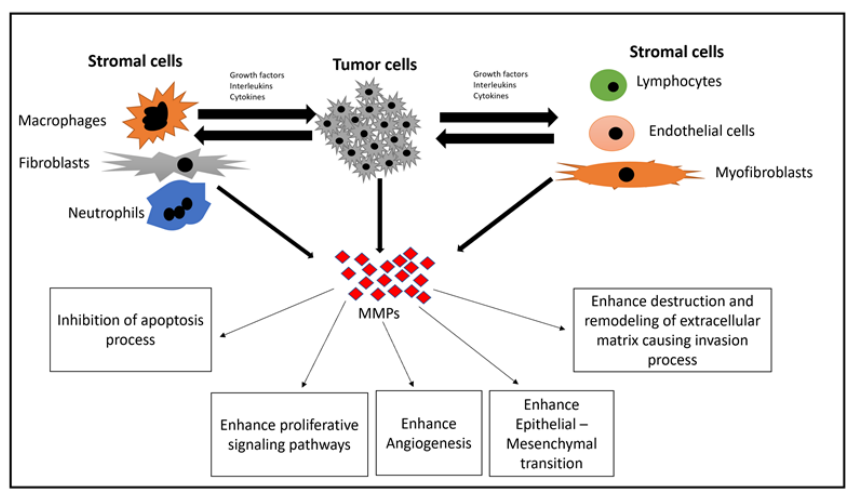

Figure 2 General roles of matrix metalloproteinases in cancer development and progression

Matrix metalloproteinases secretion within the tumour microenvironment

MMPs within the tumour tissue were thought to be exclusively released by the cancer cells. However, recent evidences have shown that these enzymes are mostly released by cancer stromal cells. ${ }^{8-11}$ These cells are originally normal tissue cells that had undergone a pathological molecular and morphological transformation within the tumour mass through paracrine communications with cancer cells in response to growth factors like vascular endothelial growth factor (VEGF), platelet-derived growth factor (PDGF), fibroblast growth factor (FGF), transforming growth factor (TGF-B) and interleukin-6 (IL-6). ${ }^{12,13}$ These cells include tumourassociated fibroblasts, pericytes, endothelial and some immune cells that actively participate with cancer cells in the oncogenic processes of progression such as in proliferation, expansion, and spread. ${ }^{8,14}$

Among the stromal cells, fibroblasts grab special attention in which under normal circumstances such as wound healing, fibroblasts cells adapt into an active form that is terminated once the function in wound healing is achieved. However, in cancer tissues, these fibroblasts are incessantly activated, and neither revert to a normal phenotype nor undergoes apoptosis..$^{15}$ In addition, these fibroblasts are found to secrete higher amounts MMPs accelerating different pathological process within the cancer tissue. ${ }^{15}$

\section{Epithelial Mesenchymal Transition:}

Epithelial mesenchymal transition (EMT) is a biological process which involves a change in 
the epithelial characteristics of cells by losing the tissue orientation and gaining a motile mesenchymal phenotype. ${ }^{19}$ This transformation is essential in many physiological functions such as in embryonic development, wound healing and in pathological conditions where cancer cells gain these migratory characteristics. ${ }^{20}$ Once at the secondary location, these cancer cells revert back to their epithelial phenotype and may remain dormant for years before starting the secondary growth. ${ }^{20}$ As shown in Figure 3, cancer cells undergoing EMT are characterized by decreased expression of epithelial markers, such as Ecadherin, claudin, ZO-1, and occludin and increased expression of mesenchymal markers such as $\mathrm{N}$-cadherin, vimentin, fibroblast-specific protein 1 , and fibronectin. ${ }^{21}$ This transition is mainly determined by the status of the ECM. When the ECM is stiff, cancer cells may retain the mesenchymal phenotype to pass through the micro spaces between the cells created through the degradation of ECM by MMPs. ${ }^{22}$

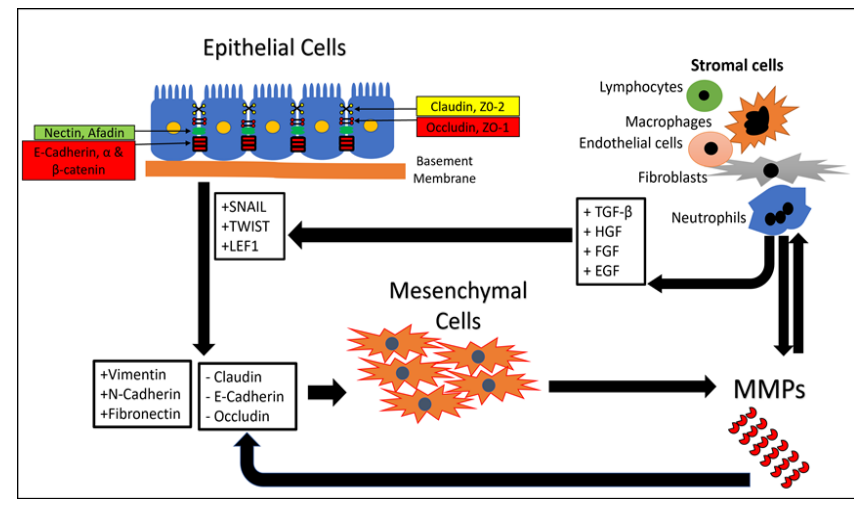

Figure 3 Effects of matrix metalloproteinases on the epithelial mesenchymal transition

MMPs enzymes participate in this complex process by activating EMT signalling pathways as shown in Figure 3 by releasing and activating growth factors such as transforming growth factor- $B$ (TGF- $B$ ), hepatocyte growth factor, fibroblast growth factor and epidermal growth factor. ${ }^{20}$ Moreover, MMPs may also proteolytically cleave E-cadherin attachment between the cells promoting dissolution of epithelial cells and releasing a bioactive fragment of E-cadherin that induces cell motility and migration. ${ }^{22}$

Cancer cells undergoing mesenchymal transformation tends to secrete higher amounts of MMPs which makes these cells less dependent on the stromal cells for their progression. ${ }^{23,}{ }^{24}$ In addition, mesenchymal cancer cells are capable of recruiting more stromal cells that would support the cancer cells with higher amounts of MMPs leading to more aggressive changes. ${ }^{22}$

\section{Invasion and metastasis}

Cancer metastasis is a complex multistep process that involves complex interactions within the tumour microenvironment. It begins with detachment of a single cancer cell or a group of cells by losing the cell attachments, and bypassing anoikis. With the help of ECM degradation, these cells may undergo an EMT and start the journey of cell migration. ${ }^{25}$ Anoikis is a type of programmed cell death in response to the loss of cellular attachment in which the mechanism of its bypass in tumour cells is largely dependent on the phenomenon of EMT to initiate the migration, invasion and metastasis journey. ${ }^{25}$ While migrating, the cancer cells would penetrate the blood or lymphatic capillaries and travel along the stream to attach within the endothelial cells and penetrate through to start the secondary growth location. ${ }^{25}$

Another MMP related mechanism playing role in the cell migratory behaviour is attributed to proteinasesactivated receptors (PARs). This can induce cancer cell migration through its activation by the proteolytic cleavage of the extracellular domain of $G$ protein-coupled receptors by MMPs. ${ }^{26}$

\section{Cancer cells proliferation}

MMPs regulate the proliferation and the growth of cancer cells through multiple mechanisms including the release of cell membrane-bound precursors of some growth factors.

Hence, MMPs increase the bioavailability of these factors in addition to its indirect action through modifying the proliferative signals from integrin molecules. ${ }^{17}$ Among these molecules, the epidermal growth factor which is one of the most important growth modulators in carcinogenesis is primarily bound to the cell membranes of cancer and stromal cells and is later released to perform its versatile functions triggered by the action of MMPs. ${ }^{27}$

In addition, MMPs may expose various cell receptors on the cell membrane of cancer and stromal cells to various cytokines and growth factors to enhance the growth and progression of cancer cells. ${ }^{24}$ The phosphorylation of the tyrosine kinase receptors 
results in activation of cancer proliferative signalling pathways such as MAPK pathway which is well-linked with cancerous proliferation. ${ }^{28}$

Another proliferative mechanism of MMPs depend on the presence of actin and other cytoskeleton proteins within the cell membrane protrusions of the invasive cancer cells. Once these protrusions are exposed and bound with soluble MMPs, the ECM will be proteolytically degraded releasing adhesive molecules such as integrins which enhances the proliferative behaviour among the cancer cells. ${ }^{24,} 29$ MMPs also releases TGF-B factor from cancer and stromal cells within the tumour mass causing an extensive proliferation of cancer cells and penetration through the ECM. This can cause the release of more cell adhesion molecules facilitating metastasis, angiogenesis, and development of immunosuppression. $^{30}$

Tumour necrosis factor alpha (TNF- $\alpha$ ) released by MMPs is one of the most important pro-inflammatory cytokines that is expressed on cancer stromal cells. When released, it may promote the growth of tumour cells by activation of nuclear factor kappa $B$ pathway (NF-kB). ${ }^{31}$

Furthermore, the proteolytic degradation of ECM by MMPs may release other membrane-bound molecules such as cadherin, integrin, superfamily of immunoglobulin (Ig-CAMs), selectins and CD44 hyaluronic receptors. These receptors actively participate in promoting cancer cells proliferation, invasion and angiogenesis. ${ }^{4}$ MMPs may also alter cancer cell cycle checkpoint control and promote genomic instability in cancer cells by affecting cell adhesions. $^{32}$

On the contrary, anti-tumour properties of MMPs were reported by activating factors capable of inhibiting tumour growth such as TGF-B or by releasing pro-apoptotic molecules such as (TNF-a, Fas ligand). ${ }^{33,} 34$

\section{Angiogenesis}

MMPs mainly influence the process of angiogenesis which is essential for tumour development and spread by two mechanisms. The first mechanism is by the proteolytic digestion of the ECM causing the release of angiogenesis-stimulating factors such as vascular endothelial growth factor. ${ }^{24}$ This stimulates the formation of new blood vessels and increase their permeability. ${ }^{24}$ The other way of contribution in angiogenesis is through breaking the basement membrane causing the escape of different stromal cells including endothelial cells to form new blood vessels in the tumour tissue. ${ }^{24}$

On the contrary, MMPs may cause inhibition of angiogenesis process by releasing angiostatins. This is mainly due to the cleavage of plasminogen by the influence on endostatin production caused by collagen XVIII cleavage. ${ }^{35}$

\section{Apoptosis}

MMPs may exhibit anti-apoptotic effects through the release of Fas ligand with inactivation of Fas death receptors and by the proteolytic release of tumourassociated major histocompatibility proteins complex class-I related factors that will prevent apoptotic events in cancer cells. ${ }^{36,37}$ MMPs may also disturb the cellular adhesion molecules resulting in inhibiting the expression of p53 protein and enhancing the expression of MDM2 proteins to interfere with the apoptosis pathway. ${ }^{38}$ in addition, MMPs can contribute to a special form of apoptosis. This is due to detachment of cancer cells from the ECM in what is called anoikis process through disruption in the integrin signalling causing activation of caspases and cell death. However, this will increase the survival of other resistance cells to apoptotic stimuli. ${ }^{39}$ In contrast, MMPs have a proapoptotic activity that is similarly linked with degradation of ECM adhesion molecules such as laminin leading to apoptosis. ${ }^{40}$

\section{CONCLUSION}

MMPs are vital factors in the development and spread of cancer cells which intensifies most of the pathological processes in cancer cells. This happens either by direct proteolytic degradation of ECM function or indirectly by releasing other factors capable of activating different cancer signalling pathways. Therefore, these enzymes can be considered as a potential target in the treatment of cancer. However, challenges may exist in this approach because these enzymes are also important in regulating many normal physiological functions. Hence, the potential treatment should be directed towards restoring the normal balanced level of these enzymes and breaking the viscous cycle 
created by the interaction between cancer and stromal cells.

\section{ACKNOWLEDGMENT}

This study was supported by the fundamental research grant scheme (FRGS17-005-0571).

CONFLICTS OF INTEREST: The authors declare no conflict of interest

\section{REFERENCES}

1. Kessenbrock, K., Wang, C.-Y., Werb, Z. Matrix metalloproteinases in stem cell regulation and cancer. Matrix Biology. 2015,44:184-90.

2. Gialeli, C., Theocharis, A. D., Karamanos, N. K. Roles of matrix metalloproteinases in cancer progression and their pharmacological targeting. The FEBS journal. 2011,278,(1):1627.

3. Pittayapruek, P., Meephansan, J., Prapapan, O., Komine, M., Ohtsuki, M. Role of matrix metalloproteinases in photoaging and photocarcinogenesis. International journal of molecular sciences. 2016,17,(6):868.

4. Bourboulia, D., Stetler-Stevenson, W. G., editors. Matrix metalloproteinases (MMPs) and tissue inhibitors of metalloproteinases (TIMPs): Positive and negative regulators in tumor cell adhesion. Seminars in cancer biology; 2010: Elsevier.

5. Kessenbrock, K., Plaks, V., Werb, Z. Matrix metalloproteinases: regulators of the tumor microenvironment. Cell. 2010,141,(1):52-67.

6. Nissinen, L., Kähäri, V.-M. Matrix metalloproteinases in inflammation. Biochimica et Biophysica Acta (BBA)-General Subjects 2014,1840,(8):2571-80.

7. Shay, G., Lynch, C. C., Fingleton, B. Moving targets: Emerging roles for MMPs in cancer progression and metastasis. Matrix Biology. 2015,44:200-6.

8. Cirri, P., Chiarugi, P. Cancer-associatedfibroblasts and tumour cells: a diabolic liaison driving cancer progression. Cancer and Metastasis Reviews. 2012,31,(1-2):195-208.

9. Park, J.-i., Lee, J., Kwon, J.-L., Park, H.-B., Lee, S.-Y., Kim, J.-Y., et al. Scaffold-free coculture spheroids of human colonic adenocarcinoma cells and normal colonic fibroblasts promote tumorigenicity in nude mice. Translational oncology. 2016,9,(1):79-88.
10. Xing, F., Saidou, J., Watabe, K. Cancer associated fibroblasts (CAFs) in tumor microenvironment. Frontiers in bioscience: a journal and virtual library. 2010,15:166. Whipple, C., Brinckerhoff, C. BRAFV600E melanoma cells secrete factors that activate stromal fibroblasts and enhance tumourigenicity. British journal of cancer. 2014,111,(8):1625-33.

11. Bremnes, R. M., Dønnem, T., Al-Saad, S., AlShibli, K., Andersen, S., Sirera, R., et al. The role of tumor stroma in cancer progression and prognosis: emphasis on carcinoma-associated fibroblasts and non-small cell lung cancer. Journal of Thoracic Oncology. 2011,6,(1):209-17.

12. Fabris, V. T., Sahores, A., Vanzulli, S. I., Colombo, L., Molinolo, A. A., Lanari, C., et al. Inoculated mammary carcinoma-associated fibroblasts: contribution to hormone independent tumor growth. BMC Cancer. 2010,10,(1):1.

13. Flach, E. H., Rebecca, V. W., Herlyn, M., Smalley, K. S., Anderson, A. R. Fibroblasts contribute to melanoma tumor growth and drug resistance. Molecular Pharmacology. 2011,8, (6):2039-49.

14. Shiga, K., Hara, M., Nagasaki, T., Sato, T., Takahashi, H., Takeyama, H. Cancer-associated fibroblasts: their characteristics and their roles in tumor growth. Cancers (Basel). 2015,7, (4):2443-58

15. Singh, D., Srivastava, S. K., Chaudhuri, T. K., Upadhyay, G. Multifaceted role of matrix metalloproteinases (MMPs). Frontiers in molecular biosciences 2015,2:19.

16. Hua, H., Li, M., Luo, T., Yin, Y., Jiang, Y. Matrix metalloproteinases in tumorigenesis: an evolving paradigm. Cell Molecular Life Sciences. 2011,68, (23):3853-68.

17. Chaudhary, A., Chaudhary, S., Ghosh, K., Nadkarni, A. Pleiotropic Roles of Metalloproteinases in Hematological Malignancies: an Update. Asian Pacific journal of cancer prevention. 2016,17,(7):3043.

18. Lamouille, S., Xu, J., Derynck, R. Molecular mechanisms of epithelial-mesenchymal transition. Nature reviews Molecular cell biology 2014,15,(3):178.

19. Biddle, A., Mackenzie, I. C. Cancer stem cells and EMT in carcinoma. Cancer and Metastasis Reviews. 2012,31,(1-2):285-93. 
20. Yeung, K. T., Yang, J. Epithelial-mesenchymal transition in tumor metastasis. Molecular oncology. 2017,11,(1):28-39.

21. Ungefroren, H., Sebens, S., Seidl, D., Lehnert, H. , Hass, R. Interaction of tumor cells with the microenvironment. Cell Communication and Signaling. 2011,9,(1):18.

22. Ala-aho, R., Kähäri, V.-M. Collagenases in cancer. Biochimie. 2005,87,(3):273-86.

23. Jabłońska-Trypuć, A., Matejczyk, M., Rosochacki, S. Matrix metalloproteinases (MMPs), the main extracellular matrix (ECM) enzymes in collagen degradation, as a target for anticancer drugs. Journal of enzyme inhibition and medicinal chemistry. 2016,31,(sup1):177-83.

24. Jung, H.-Y., Fattet, L., Yang, J. Molecular pathways: linking tumor microenvironment to epithelial-mesenchymal transition in metastasis. Clinical Cancer Research. 2015,21,(5):962-8.

25. Zigler, M., Kamiya, T., Brantley, E. C., Villares, G. J., Bar-Eli, M. PAR-1 and thrombin: the ties that bind the microenvironment to melanoma metastasis. Cancer Research. 2011,71,(21):65616.

26. Dahl, K. D. C., Symowicz, J., Ning, Y., Gutierrez, E., Fishman, D. A., Adley, B. P., et al. Matrix metalloproteinase 9 is a mediator of epidermal growth factor-dependent E-cadherin loss in ovarian carcinoma cells. Cancer Research. 2008,68, (12):4606-13.

27. Paye, A., Truong, A., Yip, C., Cimino, J., Blacher, S., Munaut, C., et al. EGFR activation and signaling in cancer cells are enhanced by the membrane-bound metalloprotease MT4-MMP. Cancer research. 2014

28. Rozanov, D. V., Hahn-Dantona, E., Strickland, D. K., Strongin, A. Y. The low density lipoprotein receptor-related protein LRP is regulated by membrane type-1 matrix metalloproteinase (MT1 -MMP) proteolysis in malignant cells. Journal of Biological Chemistry. 2004,279,(6):4260-8.

29. Oshimori, N., Oristian, D., Fuchs, E. TGF-B promotes heterogeneity and drug resistance in squamous cell carcinoma. Cell. 2015,160,(5):963 $-76$.

30. Li, C., Yang, Z., Zhai, C., Qiu, W., Li, D., Yi, Z., et al. Maslinic acid potentiates the anti-tumor activity of tumor necrosis factor a by inhibiting NF-KB signaling pathway. Molecular Cancer. 2010,9,(1):73.

31. Tlsty, T. D. Cell-adhesion-dependent influences on genomic instability and carcinogenesis.
Current opinion in cell biology. 1998,10,(5):647 $-53$.

32. Xu, J., Liu, H., Wu, Y., Gong, X., Zhou, Q., Qiao, F. Proapoptotic effect of metalloproteinase 9 secreted by trophoblasts on endothelial cells. Journal of Obstetric and Gynaecological Research. 2011,37,(3):187-94.

33. Khamis, Z. I., Iczkowski, K. A., Man, Y.-G., Bou -Dargham, M. J., Sang, Q.-X. A. Evidence for a Proapoptotic Role of Matrix Metalloproteinase26 in Human Prostate Cancer Cells and Tissues. Journal of Cancer. 2016,7,(1):80.

34. Gomes-Giacoia, E., Miyake, M., Goodison, S., Rosser, C. J. Targeting plasminogen activator inhibitor-1 inhibits angiogenesis and tumor growth in a human cancer xenograft model. Molecular cancer therapeutics. 2013,12, (12):2697-708.

35. Gialeli, C., Theocharis, A. D., Karamanos, N. K. Roles of matrix metalloproteinases in cancer progression and their pharmacological targeting. FEBS journal. 2011,278,(1):16-27.

36. Waldhauer, I., Goehlsdorf, D., Gieseke, F., Weinschenk, T., Wittenbrink, M., Ludwig, A., et al. Tumor-associated MICA is shed by ADAM proteases. Cancer Research. 2008,68,(15):636876.

37. Nigro, J. M., Aldape, K. D., Hess, S. M., Tlsty, T. D. Cellular adhesion regulates $\mathrm{p} 53$ protein levels in primary human keratinocytes. Cancer research. 1997,57,(17):3635-9.

38. Paoli, P., Giannoni, E., Chiarugi, P. Anoikis molecular pathways and its role in cancer progression. Biochimica et Biophysica ActaMolecular Cell Research. 2013,1833,(12):3481 98.

39. Gialeli, C., Kletsas, D., Mavroudis, D., Kalofonos, H., Tzanakakis, G., Karamanos, N. Targeting epidermal growth factor receptor in solid tumors: critical evaluation of the biological importance of therapeutic monoclonal antibodies. Current Medical Chemistry. 2009,16,(29):3797-804. 